\title{
Biomarkers detecting minimal residual disease in solid tumors: what do they mean in the clinical management of patients?
}

\author{
Nigel P Murray*,1 \\ ${ }^{1}$ CTC Laboratory \& Faculty of Medicine, University Finis Terrae, Santiago, Chile \\ *Author for correspondence: nigelpetermurray@gmail.com
}

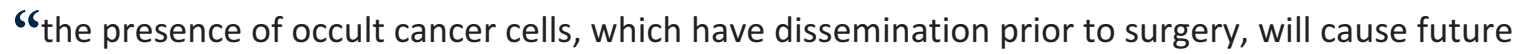 relapse and their elimination will improve disease-specific survival."”

First draft submitted: 13 September 2019; Accepted for publication: 17 September 2019; Published online: 21 October 2019

\begin{abstract}
After curative resection of the primary tumor in nonmetastatic cancer, there are three questions to be answered. First, is adjuvant therapy necessary to decrease the risk of future relapse? Second, if so, what therapy? And third, how to monitor the response?

The decision to use adjuvant therapy is based on the clinical-pathological findings of the primary tumor stage according to the TNM classification, tumor differentiation and vascular, lymphatic and/or perineural infiltration. Patients are classified according to the risk of relapse and based on the results of large double-blinded randomized trials assigned to adjuvant therapy (chemotherapy, radiotherapy, immunotherapy or hormonal therapy as monotherapy or in combination). Adjuvant therapy may be nonspecific such as chemotherapy with/without radiotherapy or specific. Specific therapy is based on the characteristics of the primary tumor; examples are trastuzumab for HER2-positive breast cancer, tamoxifen for estrogen receptor positive breast cancer [1]. In colon cancer, the determination of tumor gene status for $R A S$ and $B R A F$ is done individually, as part of next-generation sequencing, the use of microsatellite instability, or mismatch repair testing [2]. In patients with advanced non-small-cell lung cancer, $E G F R$ mutation testing, ALK testing and PD-L1 testing (category 1 based evidence), BRAF and ROS1 testing are utilized to determine specific therapies [3]. The third question is how to monitor treatment, as imaging studies are intially negative for disseminated cancer and follow-up is based on detecting treatment failure and the appearance of metastasis. The level of prostate-specific antigen after primary treatment is used as a serum biomarker to determine the need for treatment and to monitor treatment response in non-metastatic cancer only in prostate cancer.

The rational for the use of adjuvant therapy is that the presence of occult cancer cells, which have dissemination prior to surgery, will cause future relapse and their elimination will improve disease-specific survival.These occult cancer cells are termed minimal residual disease (MRD). To detect MRD there are three forms, the detection of circulating tumor cells (CTC), detection of disseminated tumor cells or micro-metastasis in bone marrow (DTC) and circulating tumor DNA. Each method has its pitfalls. CTC detection is method dependent; those based on enrichment using EpCAM such as CellSearch ${ }^{\circledR}$ do not detect EpCAM-negative CTCs, such as those that have undergone the epithelial-mesenchyme transition and can be found in benign disease [4-7]. Those based on cell size or density, such as Oncoquick ${ }^{\circledR}$, Screencell ${ }^{\circledR}$ or ISET ${ }^{\circledR}$, do not detect cell clusters or CTCs smaller than $8 \mu \mathrm{m}$. DTCs are detected in bone marrow aspirates or biopsies and identified using immunocytochemistry or RT-PCR, which is a more invasive procedure, but serious adverse events are reported in only $0.07 \%$ of cases [8]. Both CTCs and DTCs are detected with increasing frequency with higher stage nonmetastatic cancer and their presence is associated with a worse prognosis [8-12]. More recently, the circulating tumor-free DNA (tfDNA) has been used as a measure of MRD. At present, only the detection of tumor-specific mutations on cell-free DNA indicates the presence of tfDNA. In cancer patients, a major source of tfDNA is from necrotic or apoptotic tumor cells, a minor component is that secreted from viable cancer cells, however there is no form of distinguishing between tfDNA from live or dying/dead tumor cells [13].
\end{abstract}

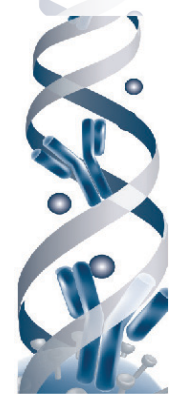


Primary tumor phenotypic and genotypic characteristics may be different from those characteristics in cells, which have disseminated and implanted in distant sites. Thus the selection of specific treatments based on the characteristics of the primary tumor may not be the most appropriate. HER2 expression in bone marrow micrometastasis has been reported to be up to $60 \%$ in comparison with the $20 \%$ expressed in primary breast tumors [14]. Similarly, HER-2-positive prostate cells are associated with resistance to androgen blockade but not diethylstilbestrol [15]. In colon cancer, $K R A S$ and $B R A F$ mutations detected in primary and metastatic cancer are used as predictive markers for anti-EGFR therapy. However, there is discordance between KRAS mutations in CTCs and primary tumors; there are more mutations in CTCs in stage I and II patients and less in stage IV patients. Furthermore, the more aggressive KRAS codon 12 mutations were more frequently detected than the KRAS codon 13 mutations in CTCs [16].

Thus the use of MRD to determine specific treatments may improve treatment results. The repeated use of MRD classification would allow changes in treatment before the appearance of metastatic disease. Changes in tumor cell sensitivity by clonal evolution or clonal selection detected during follow-up would identify patients at the risk of relapse. Serial sampling of CTCs has shown changes in the KRAS mutational status during treatment and thus predict patient response to targeted therapy [17].

This leads to another important question: when should samples be taken? CTCs are rapidly cleared from the circulation, mostly within $24 \mathrm{~h}$ [18]. There is no information on the time scale of elimination of micrometastasis. As for tfDNA, the question of how long tfDNA continues to be released from dead and dying tumour cells after treatment is not known.

More recently, differing types of MRD have been identified; those patients with CTCs are at early risk of relapse whereas those with only bone marrow micrometastasis of late relapse [12,19]. It appears that patients with CTCs have a more aggressive disease than those with only DTCs; this has been reported in esophageal cancer [20], colon cancer [12], prostate cancer [19], pancreatic cancer [11] and breast cancer [21].

This raises the interesting question of do all MRDs need to be treated? With increasingly sensitive molecular techniques to detect tumor cells, it may be that below a certain tumor load, the body is able to control tumor cell proliferation. This is the concept of dormancy. However, with time, due to changes in the tumor cell or stromal microenvironment, the cells begin to proliferate and disseminate, giving rise to metastatic diseases. This has been reported in patients with chronic myeloid leukemia post bone marrow transplant, whereby PCT-detected tumor cells are found in patients in 'complete' remission years after transplant [22]. Thus a form of active surveillance may be more effective, starting treatment when CTCs appear. This leads to the next question: do all MRDs need to be eradicated? In myeloma, treatment is continued until the paraprotein is stable, the 'plateau' phase of the disease. In patients achieving plateau, active treatment is stopped and maintenance treatment such as thalidomide, lenolidamide and/or bisphosphonates is used until the paraprotein levels start to rise. If CTCs are eradicated, leaving only bone marrow DTCs, is this equivalent to plateau phase in myeloma? Bisphosphonates have been reported to eliminate CTCs and DTCs [23-26] and as such could have a role in extending the 'plateau phase' of solid tumors.

Combined methods of detection and characterization may yield more clinically meaningful data, for example CTCs with DNA/RNA analysis permits the morphological confirmation of a tumour cell with a specific marker, for example $B R A F$ to define specific treatments. Due to the heterogeneity of tumor cells, not all tumour cells will be positive for a given marker; for example, HER-2, BRAF; thus tfDNA negative for $B R A F$ does not imply the absence of CTCs but the absence of CTCs positive for BRAF. This would also permit the identification of clonal selection post-treatment, such as ARv7 variants in prostate cancer patients and the selection of androgen blockade [27]. Single CTC sequencing shows the genetic heterogeneity of these cancer cells and has been reported to identify therapeutic resistance, multiple mutations in the ALK-independent pathways and the RTK-KRAS genes seen with crizotinib and lorlatinib resistance in patients with non-small-cell lung cancer [28].

At present, there is not a single 'gold-standard' method for the detection of CTCs and/or DTCs however, the detection of CTCs and DTCs overcomes many of the limits of surgical biopsies. Both blood and bone marrow can be repeatedly sampled for the detection of cancer cells thus detecting changes in the phenotypic and genotypic characteristics before the appearance of clinical disease.

In summary, it is important to define and understand what biomarkers mean, both positive and negative results, and the purpose of their use. Their possible role in the detection of MRD after primary curative therapy identifies high-risk patients in whom additional treatment is most likely to be beneficial. A possible role during follow-up is the early detection of treatment resistence enabling changes in treatment before the appearence of clinical metastasis. 
When detecting MRD, it is important to recognize that there are subtypes and it may not be necessary to eradicate all subtypes of MRD in order to produce clinical benefits. In the real world, health budgets do not allow high technology, high cost tests, bone marrow sampling with immunocytochemistry or detection of circulating tumor cells using density gradient differential centrifugation and immunocytochemistry or FISH may be more readily available in district hospitals. Equally important are results based on a positive/negative or yes/no decision-making choice and thus making the oncologist's job easier.

\section{Acknowledgments}

The author wishes to thank AM Palazuelos for her help in the writing of this manuscript.

\section{Financial \& competing interests disclosure}

The author has received consultancy fees from Viatar CTC Solutions, Boston, USA. The author has no other relevant affiliations or financial involvement with any organization or entity with a financial interest in or financial conflict with the subject matter or materials discussed in the manuscript apart from those disclosed.

No writing assistance was utilized in the production of this manuscript.

\section{References}

1. NCCN. 2019 guidelines breast cancer (2019). www.nccn.org/professionals/physician_gls/pdf/breast.pdf

2. NCCN. 2019 guideline colon cancer (2019). www.nccn.org/professionals/physician_gls/pdf/colon.pdf

3. NCCN. 2019 guideline non-small cell lung cancer (2019). www.nccn.org/professionals/physician_gls/pdf/nscl.pdf

4. MT Gabriel, LR Calleja, Chalopin A, Ory B, Heymann D. Circulating tumor cells: a review of non-EpCAM based approaches for cell enrichment and isolation. Clin. Chem. 62(4), 571-581 (2016).

5. Grover PK, Cummins AG, Price TJ, Roberts-Thomson IC, Hardingham JE. Ciruclating tumour cells: the evolving concept and the inadequacy of their enrichment by EpCAM based methodology for basic and clinical cancer research. Ann. Oncol. 25(8), 1506-1516 (2014).

6. Pantel K, Denéve E, Nocca D et al. Circulating epithelial cells in patients with benign colon diseases. Clin. Chem. 58(5), 936-940 (2012).

7. Murray NP, Reyes E, Badinez L et al. Circulating prostate cells found in men with benign prostate disease are P504S negative: clinical implications. J. Oncol. 2013, 165014 (2013).

8. Bain BJ. Morbidity associated with bone marrow aspiration and trephine biopsy - a review of UK data for 2004. Hematologica 9196), 1293-1294 (2006).

9. Sai B, Xiang J. Diseminated tumour cells in bone marrow are the source of cancer relapse after therapy. J.Cell Mol. Med. 22(12), 5776- 5786 (2018).

10. Tachtsidis A, McInnes LM, Jacobsen N, Thompson EW, Saunders CM. Minimal residual disease in breast cancer: an overview of circulating and disseminated tumour cells. Clin. Exp. Metastasis 33(6), 521-550 (2016).

11. Stephenson D, Nahm C, Chua T et al. Circulating and disseminated tumor cells in pancreatic cancer and their role in patient prognosis: a systemic review and meta-analysis. Oncotarget 8(63), 107223 (2017).

12. Murray NP, Aedo S, Villalon R et al. Effect of FOLFOX on minimal residual disease in stage III colon cancer and risk of relapse. Ecancermedicalscience 13, 935 (2019).

13. Yamada T, Matsuda A, Koizumi M et al. Liquid biopsy for the management of patients with colorectal cancer. Digestion 99(1), 39-45 (2019).

14. Gebhardt F, Zanker KS, Brandt B. Differential expression of alternatively spliced c-erb-2 mRNA in primary tumors, lymph node metastases and bone marrow micrometastasis from breast cancer patients. Biochem. Biophys. Res. Commun. 247(2), 319-323 (1998).

15. Murray NP, Badinez L, Dueñas RR, Orellana N, Tapia P. Positive HER-2 protein expression in circulating prostate cells and micrometastasis, resistant to androgen blockade but not diethylstibestrol. Ind. J. Urol. 27(2), 200 (2011).

16. Liu Y, Meucci S, Sheng L, Keilholz U. Meta-analysis of the mutational status of circulation tumor cells and paired primary tumor tissues from colorectal cancer patients. Oncotarget 8, 77928-77941 (2017).

17. Kalikaki A, Politaki H, Souqlakos J et al. KRAS genotypic changes of circulating tumor cells during treatment of patients with metastatic colorectal cancer. PLoS ONE 8(44), 77928 (2014).

18. Murray NP, Reyes E, Orellana N, Fuentealba C, Dueñas R, Jacob O. Expression of P504S and matrix metalloproteinase-2 in circulating prostate cells disseminated as a result of transrectal ultrasound guided biopsy as determined by immunocytochemistry: clinical implications. Arch. Esp. Urol. 68(5), 474-481 (2015).

19. Murray NP, Aedo S, Fuentealba C et al. Subtypes of minimal residual disease, association with Gleason score, risk and time to biochemical failure in pT2 prostate cancer treated with radical prostatectomy. Ecancermedicalscience 13, 934 (2019). 
20. Konczalla L, Ghadban, Effenberg KEet al. Prospective comparison of the prognostic relevance of circulating tumor cells in blood and disseminated tumor cells in bone marrow of a single patient's cohort with esophageal cancer. Ann. Surg. (2019). doi.org.10.1097/SLA (Epub Ahead of Print)

21. Takeyama H, Shimada T, Kinoshita S, Uchida K. Usefulness of CTC and DTC-BM detection for adjuvant therapy effects and prognosis prediction in early breast carcinoma: results of 8-11 years of follow-up evaluation. Ann. Surg. Oncol. 24(5), 1227-1233 (2017).

22. Serrano J, Roman J, Sanchez Jet al. Molecular analysis of lineage specific chimerism and minimal residual disease by rt-PCR of p210(BCR-ABL) and p190 (BCR-ABL) after allogeneic bone marrow transp'lantation for chronic myeloid leukemia: increasing mixed myeloid chimerism and p190 (BCR-ABL) detection precede cytogenetic relapse. Blood 95(8), 2659-2665 (2000).

23. Ide H, Lu Y, Tanaka Tet al. Circulating tumor cell count during zoledronic acid treatment in men with metastatic prostate cancer: a pilot study. Prostate Int. 2(3), 147-151 (2014).

24. Diel IJ, Jaschke A, Solomayer EFet al. Adjuvant oral clodronate improves overall survival of primary breast cancer patients with micrometastasis to bone marrow: a long-term follow-up. Ann. Oncol. 19(12), 2007- 2011 (2008).

25. Rack B, Juckstock J, Genss EM et al. Effect of zoledronate on persisting isolated tumour cells in patients with early breast cancer. Anticancer Res. 30(5), 1807-1813 (2010).

26. Banys M, Solomayer EF, Gebauer G et al. Influence of zoledronic acid on disseminated tumour cells in bone marrow and survival: results of a prospective clinical trial. BMC Cancer 13(1), 480 (2013).

27. Sepe P, Verzoni E, Miodini P et al. Could circulating tumor cells and ARV7 detection improve clinical decisions in metastatic castration resistant prostate cancer? The Istituto Nazionale del Tumori (INT) experience. Cancers 11(7), 980 (2019).

28. Pailler E, Fangeroux V, Oulhen $\mathrm{M}$ et al. Acquired resistance mutations to ALK inhibitors identified by single circulating tumor cell sequencing in ALK-reaaranged non-small cell lung cancer. Clin. Cancer Res. 25(22), 6671-6682 (2019). 